\title{
Vorwort
}

Die vorliegende Ausgabe enthält den Text der Kirchengeschichte des Eusebius mitsamt dem Buch über die palaestinischen Märtyrer so, wie er in meiner großen Ausgabe konstituiert ist, deren am Rand vermerkte Seiten- und Zeilenzahlen im Apparat beibehalten sind. Dieser gibt vollständig nur die Änderungsvorschläge, die von mir oder anderen. sei es zu dem Text des Euseb selbst gemacht sind, sei es zu den Exzerpten, die von Euseb oft aus recht verderbten Exemplaren und nicht sehr sorgfältig ausgehoben sind, ferner die Bemerkungen, die sich auf die noch in den Hss erkennbare Differenz der von Euseb veranstalteten Ausgaben seines großen Werkes beziehen. Dagegen sind die Angaben der handschriftlichen Varianten stark reduziert, besonders bei Bibelstellen und in Exzerpten, die aus noch erhaltenen Texten genommen sind. Für kritische Untersuchungen reicht die Ausgabe also nicht aus; sie soll den Gelehrten, der sie als Handausgabe liest, den Studenten, der sie sich für Seminarïbungen anschafft, nur vorläufig über die Fundamente der Rezension orientieren: da der Text des Euseb auf einer in verschiedener Verzweigung hoch hinaufreichenden Ï̈berlieferung beruht und infolgedessen in einziger Weise fest und sicher ist, wird man mit der kleinen Ausgabe in sehr vielen Fällen auskommen.

Die sieben, genauer sechs Hss [ATERBDM], auf denen der Text beruht - denn $\mathrm{R}$ ist streng genommen entbehrlich - sowie die Versionen $[\boldsymbol{\Sigma} \boldsymbol{\Lambda}]$, sind auf S. VI aufgezählt. Es kann von ihnen weder ein Stammbaum gezeichnet. noch die eine oder andere als die "beste" angepriesen werden; jede einzelne ist reich an Fehlern und Interpolationen und alle kontrollieren sich so untereinander, daß allen Mängeln der einzelnen zum Trotz ein vorzüglicher Text herauskommt. Denn es ist von der KG nicht, wie so oft, nur ein einziges, alle mißlichen Zufälligkeiten der Überlieferung konservierendes Exemplar aus dem Altertum ins Mittelalter hinübergerettet, sondern verschiedene, z. T. dicht an den Schriftsteller heranreichende Rezensionen haben sich teils direkt in Handschriften und Handschriftengruppen. teils indirekt durch die in jene eingedrungenen 
Korrekturen erhalten. So bietet die Geschichte des Textes der KG, wie sie auf den Konkordanzen und Diskrepanzen der Hss und Versionen aufgebaut werden kann, ein bis zur Verwirrung buntes Bild, das sich im Auszug schwer darstellen läßt; ich muß dafür auf meine ausführlichen Darlegungen im III. Bande der großen Ausgabe verweisen und beschränke mich hier auf ganz weniges.

$\operatorname{BDM} \Sigma \boldsymbol{\Lambda}$ stellen die Ausgabe "letzter Hand" dar, mit der Euseb nach der Katastrophe des Licinius seine Tätigkeit an der KG abschloß. Wie das Schlußkapitel hinzugefügt wurde, so hatten andere, größere und kleinere, Partien gestrichen werden müssen, sonderlich, doch nicht ausschließlich um der damnatio memoriae des Licinius willen. Diese Partien sind, wenn auch schwerlich vollständig, aus Exemplaren der früheren Ausgabe in solche der letzten eingetragen, ferner wurde die allerdings des Anfanges und Schlusses beraubte Schrift der palaestinischen Märtyrer nach ihrer ersten Ausgabe hinzugeschrieben. Ein so korrigiertes Exemplar liegt der Gruppe ATER zugrunde; wenn einer dieser Nachträge, nicht die übrigen, sich auch in $\mathrm{M}$ findet, so hängt das damit zusammen, daB in späterer Zeit einmal ein Vorfahr von II aus TER korrigiert ist, wie sich aus der Capitulatio und vielen Lesungen ohne weiteres ergibt.

Selbstverständlich spiegelt sich die eben geschilderte, am Bestande des Werkes sofort zu erkennende Spaltung der Überlieferung auch in zahllosen einzelnen Lesungen wider; wenn auch BDM $\Sigma \boldsymbol{A}$ in der Mehrzahl der Fälle aus irgendwelchen Gründen den Vorzug verdienen, so hat doch auch die andere Gruppe nicht selten das Richtige erhalten; oft ist eine bestimmte Entscheidung überhaupt nicht möglich.

Aus diesen Hauptgruppen lösen sich andere ab, die nicht so hoch hinaufreichen, unter Umständen aber auch Richtiges erhalten oder auflesen: so die Gruppe TER, neben der, da $\mathrm{T}$ wiederholt in alter Zeit durchkorrigiert ist, $\mathrm{T}^{\circ} \mathrm{ER}$ steht, wie $\mathrm{AT}^{ } \mathrm{ER}$ neben ATER. Als junge, meist unzuverlässige Zusammenstellungen sind zu bezeichnen $\mathrm{AT}^{1}$ - $\mathrm{AT}^{\circ}$ kommt nicht vor -, DM, BD. Bei dieser Gruppe ist besondere Vorsicht geraten, denn in ihr steckt sowohl eine junge, stark interpolierende Rezension als die alte, stets beachtenswerte Gruppe BDM, dann nämlich, wenn M dieser Gruppe aus irgendwelchen Gründen untreu geworden ist.

In jeder Hs ist eine Summe sehr verschiedenartiger Über[ieferungen zusammengeflossen, die sich einigermaßen sondern lassen nur nach den Gruppen, denen eine Hs jeweilen sich anschließt: schon dadurch ist eine Prädizierung der einzelnen Hss nach ihrem Wert 
unmöglich. Aber zu beachten ist, daß A und M sowohl die häufigen Gruppen TER und BD durch ihren Hinzutritt zu einer ganz anderen Dignität emporheben, als auch die einzigen Hss sind, die oft mit wirklichen Lesarten - nicht Schreibfehlern - allein stehen. Wenn diese auch meist verworfen werden müssen, so ist doch nicht zu bestreiten, daB namentlich $\mathrm{A}$ nicht ganz selten allein den ursprünglichen Text erhalten hat.

Wer endlich mit der Erwartung an das Studium der Übersetzungen herangeht, daß in ihnen eine Fülle von Verbesserungen des in den Hss überlieferten Textes zu finden sei, wird herbe enttäuscht sein; nur sehr selten haben sie etwas erhalten, das in allen Hss verloren war. Ihre Bedeutung ist darum nicht geringer; sie liegt nicht in den Lesarten, die sie hinzubringen, sondern in denen, die sie bestätigen und dadurch hoch über das Alter der sie vertretenden Hss hinaufdatieren.

Einen vollständigen Ü̉berblick über all diese Mannigfaltigkeit kann der reduzierte Apparat dieser Ausgabe nicht verschaffen: die Fehler z. B. von AT', DM, BD mußten der großen Mehrzahl nach weggelassen werden. Auch das Schwanken in der Wortstellung zwischen BDM und ATER ist meist nicht notiert, ebensowenig $\delta^{\prime} \circ v^{\nu} \nu \sim \gamma^{\prime} \circ v^{\nu} \nu$ das Fehlen oder Setzen des Artikels vor $\overline{\boldsymbol{9} \sigma}$ und $\overline{\chi \sigma}$ u. ä. m. Dagegen sind die Varianten, wenn sie zu einer Stelle überhaupt notiert sind, stets vollständig gegeben: in diesen Fällen kann auch ex silentio geschlossen werden.

Die Capitulatio rïhrt, worauf ich besonders aufmerksam mache, von Euseb selbst her und ist keineswegs wertlos; die späteren Wucherungen habe ich weggelassen. Dagegen sind die Rückverweisungen durch die an den Rand gesetzten griechischen Ziffern nicht ursprünglich, aber der handschriftlichen Überlieferung entlehnt.

Unleugbar würde ein Index den Gebrauch der Ausgabe erleichtern; er hätte sich auch durch Kürzung des im III. Bande der großen Ausgabe enthaltenen ohne große Mühe herstellen lassen. Aber man wird es mir hoffentlich nicht verübeln, wenn ich mich zu dieser Verstümmelung meines eigenen mühseligen Werkes nicht habe entschließen können, um so weniger, als dieser Band daraufhin angelegt ist, neben der kleinen Ausgabe gebraucht zu werden, und gesondert verkauft wird.

Freiburg i. B., Ostern 1914

\section{E. Schwartz}

